\title{
Die Oberflächen-Rauhigkeit mikrotomüberschnittenen Douglasienholzes
}

\section{Hecker, G. Becker}

Institut für Forstbenutzung der Universität Göttingen, Büsgenweg 4, D-37077 Göttingen

Subject Surface roughness of tangential microtome-cut Douglas-fir was measured in early and late wood, in sapwood and heartwood. Its relationship to several wood properties and to the roughness of veneers of the same material was investigated.

Material und Methoden Zielsetzung war, die Abhängigkeit der Oberflächen-Rauhigkeit mikrotomüberschnittenen Douglasienholzes von ausgewählten Holzeigenschaften zu untersuchen und mit der Rauhigkeit von Schälfurnier-Oberflächen zu vergleichen. Dazu wurden von breit- und engringigen Stammscheiben 54 jähriger Douglasien würfelförmige, auf $u=12 \%$ konditionierte Probekörper mit dem Mikrotom in Faserrichtung tangential überschnitten. Auf reinen Frühund Spätholzpartien erfolgten insgesamt 1150 Messungen nach dem Tastschnittverfahren, und die Oberflächen-Rauhigkeit wurde u.a. mittels der Kennwerte $R_{2}$ und $R_{\max }$ (DIN 4768) beschrieben. Weiterhin wurden aus dem gleichen Ausgangsmaterial Schälfurniere industriel hergestellt und deren Rauhigkeit bestimmt. Die Taststrecke betrug $L_{T}=$ $17,5 \mathrm{~mm}$ bei einer Grenzwellenlänge von $\lambda_{c}=2,5 \mathrm{~mm}$.
Resultate 1. Zwischen Splint und Kern bestehen grundsätzlich keine Unterschiede in der Rauhigkeit. 2. Dagegen ist die durchschnittliche Oberflächen-Zerklüftung $\left(R_{z}\right)$ im Frühholz 2 bis 3 mal höher als im Spätholz, bedingt durch den unterschiedlichen Zellaufbau. 3. Die Jahrringbreite übt nur mittelbar über die Frühholzbreite einen positiv gerichteten Einfluß auf die Rauhigkeit aus. 4. Während die Rauhigkeit im Frühholz mit steigender Frühholzdichte geringer wird, weist die Rauhigkeit im Spätholz keine Abhängigkeit zur Spätholzdichte auf. 5. Die Spitzen-Rauhigkeit $\left(R_{\max }\right)$ ist positiv mit dem Dichtekontrast korreliert. 6. Der Unterschied der an mikrotomierten Oberflächen und zum Vergleich an Schälfurnieren des gleichen Materials erhobenen Oberflächen-Rauhigkeit (Tabelle 1) zeigt den Bearbeitungseinfluß, aber auch Perspektiven für technische Verbesserungen bei der Furnierherstellung.

\begin{tabular}{|c|c|c|c|c|c|c|c|c|c|}
\hline & \multirow[b]{2}{*}{$n$} & \multirow{2}{*}{$\begin{array}{l}\text { Frühholz } \\
\overline{\bar{X}}\end{array}$} & \multirow{2}{*}{$\frac{\text { Splint }}{s}$} & \multicolumn{2}{|c|}{ Spätholz } & \multirow{2}{*}{$\begin{array}{l}\text { Frühholz } \\
\overline{\bar{X}}\end{array}$} & \multirow{2}{*}{$\frac{\text { Kern }}{s}$} & \multicolumn{2}{|c|}{ Spätholz } \\
\hline & & & & $\bar{X}$ & $s$ & & & $\bar{X}$ & $s$ \\
\hline Mikrotom & 288 & 40,9 & 5,8 & 19,4 & 4,9 & 39,5 & 6,5 & 19,5 & 4,5 \\
\hline Schälfurnier & 50 & 122,2 & 19,2 & 59,7 & 16,2 & 113,2 & 34,0 & 67,6 & 15,3 \\
\hline
\end{tabular}

Tabelle 1. Vergleich der Rauhigkeit von Schälfurnier und mikrotomiertem Vollholz der Douglasie anhand des Kennwertes $R_{z}$ (DIN 4768; in $\mu \mathrm{m}$ ) 